\title{
Age and sex-associated variation in the microbiome of a rhesus macaque social group
}

Mareike Cora Janiak

Michael Montague

Catalina Villamil

Michala Stock

Amber Trujillo

Allegra DePasquale

Joseph Orkin

Samuel Bauman Surratt

Olga Gonzalez

Michael Platt

Melween Martinez

Susan Antón

Gloria Dominguez-Bello

Amanda Melin

James Higham

\section{Video Byte}

Keywords: aging, non-human primates, genital microbiome, oral microbiome, gut microbiome, sex differences, rhesus macaques, microbiota, Microbiome

Posted Date: October 15th, 2021

DOl: https://doi.org/10.21203/rs.3.rs-969895/v1

License: (c) (i) This work is licensed under a Creative Commons Attribution 4.0 International License. Read Full License 


\section{Abstract}

Our bodies are home to millions of microscopic organisms - our microbiome. While these microbes have many important functions that maintain our health and well-being, our microbiome changes as we age, which can cause infection or inflammation. Understanding these changes in humans is difficult due to our long lifespan and confounding factors such as healthcare and diet. A recent study used an animal model - free-ranging rhesus macaques - to better understand the changes that occur in our microbiomes with age, using a cross-sectional dataset of oral, rectal, and genital swabs collected from 105 macaques belonging to one social group. In contrast to adult macaques, researchers found that infant gut microbial communities had higher abundances of Bifidobacterium and Bacteroides, consistent with a milk-rich diet. The genital microbiome varied substantially between males and females, and while penile microbiomes changed with age, vaginal microbiomes did not. Oral microbiome composition changed with age, with infants exhibiting the most distinct microbiome. While longitudinal studies are needed to confirm these changes in the same animals throughout their lives, this study highlights the age-related microbial changes that occur in primates and identifies rhesus macaques as an ideal model to help prevent age-related diseases in humans. 